\title{
Carta al editor en relación al artículo: "Estrategia de uno en uno para mejorar la técnica correcta de higiene de manos"
}

\author{
Letter to the editor in relation to the \\ article: "One-on-one strategy to improve \\ the correct hand hygiene technique".
}

Estimado Editor:

Hemos leído con interés el artículo titulado: "Estrategia de uno en uno para mejorar la técnica correcta de higiene de manos" de Orozco Hernández y colaboradores, publicado en el volumen 38, número $5 .{ }^{1}$ Aunque consideramos que el estudio puede ser importante en la búsqueda de estrategias para mejorar la técnica de lavado de manos en personal de salud, nos gustaría expresarle algunas dudas que surgen y que requieren clarificación.

En primer lugar, nos llamó la atención la manera en la que se realizaron las tres etapas, en las cuales el personal de la unidad de vigilancia epidemiológica interactuó con la población en estudio para evaluar el correcto lavado de manos. En el manuscrito no queda claro quiénes eran los miembros de dicho sistema de vigilancia epidemiológica. Esto puede ser importante, porque si son más de una persona haciendo la vigilancia, debería expresarse información acer- ca de la concordancia y calibración de dichos evaluadores. Más aún, hubiese sido ideal que aquél que entregue la intervención (educación) debiera ser distinto del evaluador sobre el uso de la técnica, ya que el sesgo del observador podría estar presente. ${ }^{2}$ Adicionalmente, no se especifica en qué momento del contacto entre evaluador y participante se realizó la evaluación de la técnica de lavado de manos.

En segundo lugar, la manera en que midieron el lavado de manos en tres etapas no fue la más adecuada, ya que existen maneras más objetivas de hacerlo. Según Marra AR y Armelino D, existe una manera más precisa de observar un adecuado lavado de manos, utilizando cámaras de vigilancia que se encontraban al costado de los lavaderos y dispensadores de jabón. ${ }^{3}$ Se evaluó en tiempo real la técnica de lavado de manos tanto en hospitalización como en la Unidad de Cuidados Intensivos, este proceso fue realizado por personal capacitado en lavado de manos y hubieron otros encargados en realizar la retroa- 
limentación respecto a su técnica y la manera correcta de realizarla. ${ }^{4,5}$

Finalmente, aunque el número de participantes excluidos del estudio por data no completa durante el seguimiento es pequeño, debería primar el principio de "intención de tratar" (intent to treat $)^{6}$ muy común en los trabajos experimentales, especialmente en los ensayos clínicos, pero también en otros tipos.

\section{REFERENCIAS}

1. Hernandez HG. One-to-one training to increase correct hand hygiene technique. Act Pediatr Mex. 2017;38(05):299307.

2. Manterola C, Otzen T. Los Sesgos en Investigación Clínica. Int. J. Morphol, 2015;33(3):1156-1164.
3. Marra AR, D'Arco C, Bravim BA. Controlled trial measuring the effect of a feedback intervention on hand hygiene compliance in a step-down unit. Infect Control Hosp Epidemiol 2008;29:730e735.

4. Armellino D, Hussain E, Schilling ME. Using high-technology to enforce low-technology safety measures: the use of third-party remote video auditing and real-time feedback in healthcare. Clin Infect Dis 2012;54:1e7.

5. Armellino D, Trivedi M, Law I. Replicating changes in handhygiene in a surgical intensive care unit with remote video auditing and feedback. Am J Infect Control 2013;41:925e927.

6. Manterola C, Otzen T. Experimental Studies 1st Part. Clinical Trial. Int. J. Morphol. 2015;33(1):342-349.

Carpio-Rodríguez A Mercado-Gonzáles S Escuela de Medicina, Universidad Peruana de Ciencias Aplicadas. sofimg2311@gmail.com 